\title{
Configurational effects on the enumeration of dots: Counting by groups
}

\author{
MICHIEL P. van OEFFELEN and PETER G. VOS \\ University of Nijmegen, Nijmegen $6500 \mathrm{HE}$, The Netherlands
}

\begin{abstract}
The processing time for quantifying numerosity of two-dimensional dot patterns was investigated as a function of both number of dots and relative proximity between dots. A cluster algorithm (CODE) was first developed as a formal model of how human subjects organize neighboring dots into groups. CODE-based predictions of grouping effects on number processing latencies were then tested with patterns consisting of $n$ dots (range $n=13-23$ ). The results largely confirmed CODE-based predictions and thereby indicated that large collections of dots are preferably counted by groups. Small $(n \leqslant 5)$ groups are subitized and their partial results are summed to a running total. Based on criteria other than dot proximity, large $(n>5)$, proximity-based groups are subdivided into smaller groups of two or three dots, which are again subitized.
\end{abstract}

It is intuitively clear that the time required to ascertain the exact number of objects in a given set depends not only on the magnitude or size of the set, but also on the arrangement of objects (i.e., their patterning). This holds in particular for numbers beyond the span of immediate apprehension, also called subitizing (after Kaufman, Lord, Reese, \& Volkman, 1949). Thus, a set of 20 objects is likely to take much more processing time when it is patterned according to a uniformly spaced row than when it is a two-dimensional pattern segmented into five groups of 4 objects each. The row can hardly be enumerated by a procedure other than counting one by one. The other pattern can be processed by subitizing the five groups and summing those results in four steps of simple addition. Moreover, counting of the row is easily hampered by lateral interference of neighboring dots during the processing of a particular dot, whereas such difficulty is not so apparent with the second pattern.

Although the interactive effect of number and patterning on the processing of visual number has been explicitly noted by earlier authors (see Woodworth \& Schlosberg, 1954, chap. 4), remarkably few later studies have paid attention to it. Rather, the patterning factor was purportedly canceled out by the use of several differently scrambled dot patterns for the representation of one and the same number. However, even so-called random dot patterns may be perceived as being segmented into groups. Such a perception may be caused by, among other factors, relative differences in interdot proximity. In some of these stimuli, moreover, the size of the groups will be within the subitizing span, whereas others will exceed this range. It seems dubious, there-

This research was supported by a grant from the Netherlands Organization for the Advancement of Pure Research (Zuiver Wetenschappelijk Onderzoek). fore, to base conclusions about the latency-numerosity function solely on measures of central tendency, without discussing or even specifying dispersion values (e.g., Beckwith \& Restle, 1966; Klahr, 1973; Klahr \& Wallace, 1976).

At least one latency experiment (Klahr, 1973) supports our assertion that grouping of even random dot patterns must be more adequately controlled for than it has been in past research. Briefly summarized, Klahr wanted to know whether number-naming latencies were affected by pattern density or visual angle of a display of dots in the range of $n=1-20$. He presented stimuli under two visual conditions, one in which the dots were randomly clustered in the center of the display ("inner condition") and the other in which they were randomly spread out in the periphery of the display ("outer condition") (Figure 1). Differences between mean latencies for outer and inner conditions were calculated, and it was concluded that no consistent relationship emerged with respect to the values of $n \geqslant 7$. A closer inspection of Klahr's data, however, showed that, with two exceptions, the outer condition stimuli were processed faster than the stimuli of the inner condition. This can be explained by the higher probability of the "outer" stimuli to be perceived as a set of proximity-based, small, and subitizable clusters. The "inner" stimuli much less favor such segmentation; in other words, they are predominantly seen as a cluster the size of which remains beyond the subitizing range. Klahr's results indicate that stimulus groupability affects number-naming latencies. This effect raises the questions of whether and how one can predict the course of latencies for numbers larger than, say, $n=6$ as a function of the interaction between set size and patterning.

Bourdon (1908) was probably the first experimenter to manipulate the factor of patterning in onedimensionally arranged dot figures. He concluded from 

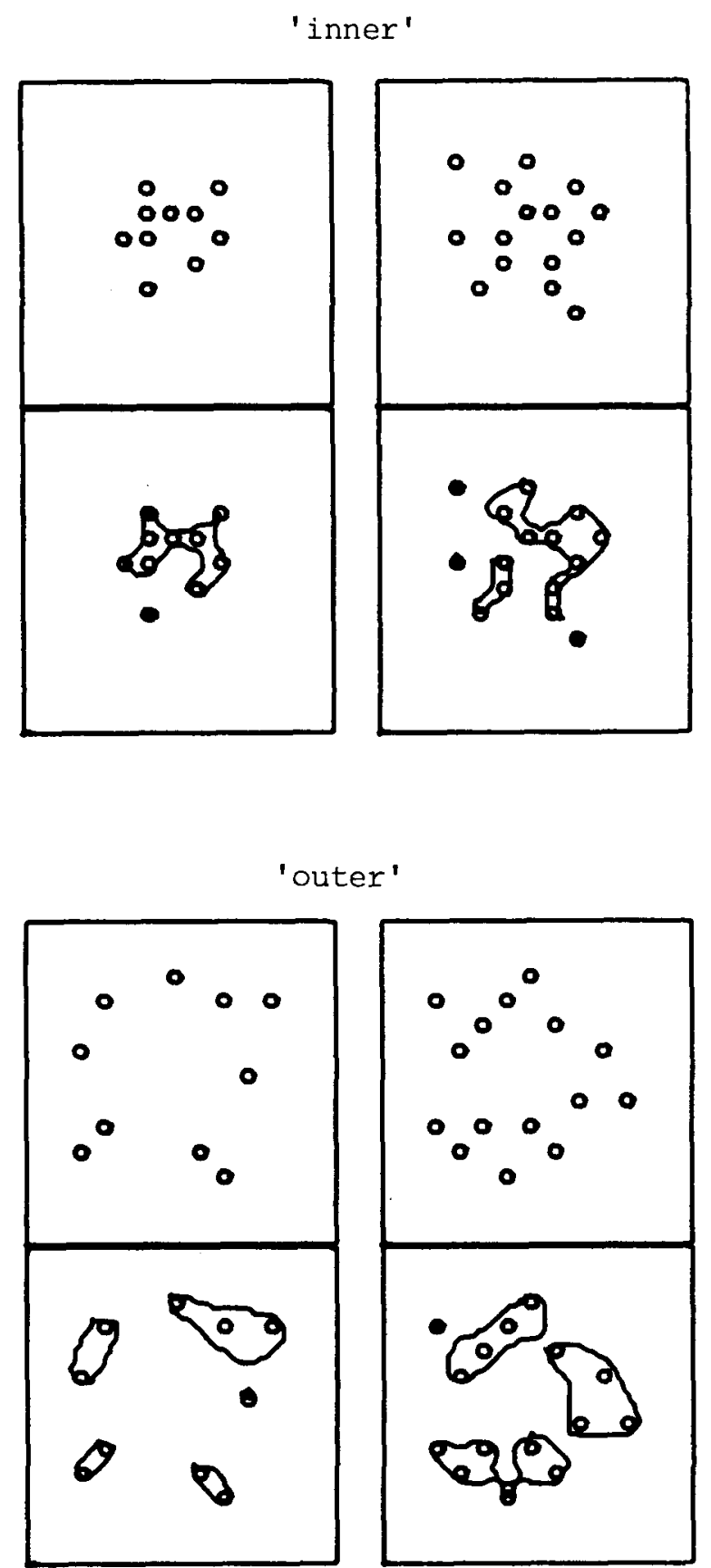

Figure 1. A few typical patterns in the "inner" and "outer" conditions, used by Klahr (1973), are presented. CODEpredicted clusters are shown below these patterns.

experimental results that the perception of number presupposes at least two psychological operations: (1) the perception of units of which the total number of dots is composed and (2) the grouping of these units. Much later, Atkinson, Cambell, and Francis (1976a, 1976b) varied the interdot interval along an array of dots so that a number of dots were clearly seen as a single entity whereas other dots were seen in another group. Atkinson et al. related the "chunking" of infor- mation to the bandwidth of channels tuned to a particular size or spatial frequency. In an experiment, they showed that if two parts of a dotted figure were discriminable by an intergroup space of three times the interdot interval, then accurate counting within each subgroup up to a maximum of four could take place.

Also, Beckwith and Restle (1966) manipulated the factor of patterning, and they successfully showed that the interaction in question does exist. In the discussion of their experiments, they referred to Gestalt-theoretical rules of proximity, good continuation, and similarity as a basis for understanding pattern-number interaction. Although these rules have been generally accepted as important guidelines for understanding how visual forms of patterns are perceptually organized (Zusne, 1970), their predictive power remains weak as long as one cannot formulate them in operational terms that allow for quantitative predictions instead of informal ad hoc demonstrations.

The present study is a first attempt to develop a predictive model about the interaction between patterning and number of dots during the processing of numbers in the range beyond the subitizing span. We first propose an algorithm, called CODE, that is a formal description of how proximity-dependent grouping takes place in stimuli like those exemplified in Figure 1. After an experimental test of the perceptual validity of CODE, we elaborate a number of equations for number response latencies as a function of both set size and within-set groupability. The subsequently derived hypotheses are then tested in a latency experiment.

\section{CODE : A CONTOUR-DETECTING CLUSTER ALGORITHM}

According to Gestalt theory, the perceptual interpretation of a figure is governed by, among other principles, the principle of proximity: A person tends to group those elements of a figure that are close to each other. Attempts to formalize grouping according to proximity have been made since the early 1960s, when computer facilities became progressively available. However, these various cluster algorithms are less appropriate for studies in visual pattern perception. First, many of these algorithms have arbitrary stop rules. That is, the level of clustering that optimally reflects perceptual grouping is determined largely by intuition. Second, most of these algorithms were developed to handle very large sets of elements and are not well suited to finding clusters in small sets.

Perceptual psychologists (e.g., Marr, Note 1; Zucker, Rosenfeld, \& Davis, Note 2) have emphasized the idea that there exist early stages in visual perception that are not determined by the perceiver's knowledge of the semantic aspects of the visual input. Marr (Note 1) argues that a great deal of information may in fact be extracted from an image by means of knowledge-free 
techniques. Grouping considerations based on orientation, proximity, gray level, color, and so on, provide a computation of a primal description of the image (the so-called "primal sketch"). This primal sketch should be a result of a purely data-driven and context-free algorithm. The image formation may be described mathematically in terms of degradation operators or spread functions that characterize the optical channel.

The method proposed in this study involves a procedure in which a binormal distribution function is superposed on each element of a dot figure. This function should represent the strength with which an element operates upon its surroundings. The concept of neighborhood regarding relative proximity is expressed by the width of the spread function. This dispersion is fixed by the distance between each element and its nearest neighbor. Clusters are then formed in regions in which the sum of the partial functions reaches a threshold value.

The simplest case of clustering includes a set of only two dots. We can represent the dots (labeled $p_{1}$ and $p_{2}$ ) in one dimension with coordinates $x_{1}$ and $x_{2}$, respectively. The basic assumption is that two dots are always grouped because the distance, $d$, between exactly two dots can be related only to itself. That is, the clustering strength between only two dots is optimal no matter how large the distance is between those points. For the sake of simplicity, any restriction to the boundaries of the stimulus field is left out of consideration. In order to describe the clustering of $p_{1}$ and $p_{2}$, a normal distribution function, $\mathrm{f}_{\mathrm{p}_{\mathrm{i}}}(\mathrm{x})=\exp -\left[\left(\mathrm{x}_{\mathrm{i}}-\mathrm{x}\right)^{2} / 2 \mathrm{~s}_{\mathrm{i}}^{2}\right]$, which is further defined as $\left[x_{i}, s_{i}\right]$, is superposed on each of the two dots: $f_{p_{1}}(x)=\left[x_{1}, s_{1}\right]$ and $f_{p_{2}}(x)=\left[x_{2}, s_{2}\right]$, as is illustrated in Figure 2. The strength with which a dot influences its surroundings is reflected by the property of the normal function: The effect is maximal at its own

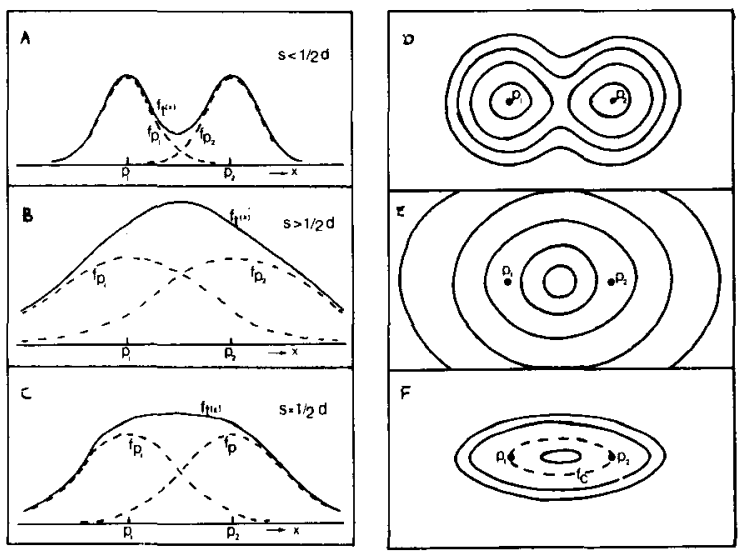

Figure 2. The functions $f_{p_{1}}(x), f_{p_{2}}(x)$, and $f_{t}(x)$ are plotted as a function of $x$ for $s \leqslant 1 / 2 d, s>1 / 2 d$, and $s=1 / 2 d$, respectively (Figures $2 \mathrm{a}, 2 \mathrm{~b}$, and $2 \mathrm{c}$ ). The two-dimensional representations are plotted in Figures $2 \mathrm{~d}, 2 \mathrm{e}$, and $2 \mathrm{f}$, respectively. The set $f_{c}$ of points $(\bar{x})$ for which it holds that $f_{t}(\bar{x})=f_{0}$ is shown in Figure 2f. position and monotonically decreasing to zero for its peripheral surroundings. The range of a point's influence is fixed by the standard deviation or dispersion, $s$. This parameter must be selected to yield an optimal representation of clustering strength. In relation to $p_{1}$ and $p_{2}$, it is clear that $s_{1}$ equals $s_{2}$ from the point of view of symmetry, so that $s_{1}=s_{2}=s$. When the dispersion is given a very small value with respect to the distance, $d$, between $p_{1}$ and $p_{2}$, the summed function $f_{t}(x)=$ $\left[\mathrm{x}_{1}, \mathrm{~s}\right]+\left[\mathrm{x}_{2}, \mathrm{~s}\right]$ will show a minimum just between $\mathrm{p}_{1}$ and $\mathrm{p}_{2}$ (Figure $2 \mathrm{a}$ ). In that case, $\mathrm{f}_{\mathrm{t}}(\mathrm{x})$ underrepresents the assumed strength of clustering. However, when the dispersion is large in comparison with $\mathrm{d}$, the resultant function $f_{t}(x)$ will show a sharp peak between $p_{1}$ and $p_{2}$. In this case, $f_{t}(x)$ overrepresents the clustering of $\mathrm{p}_{1}$ and $\mathrm{p}_{2}$ (Figure 2b). It was decided, therefore, to take that value for $s$ in which $f_{t}(x)$ just reaches a maximum, which emerges when $s=1 / 2 d$ (Figure $2 c$ ).

The three clustering representations depicted in Figures $2 a, 2 b$, and $2 c$ are in one-dimensional form. Expanding them to a two-dimensional form yields a description of the clustering in terms of contours (see Figures $2 \mathrm{~d}, 2 \mathrm{e}$, and $2 \mathrm{f}$ ), which are isolines of the twodimensional function $f_{t}(\bar{x})$. In order to distinguish between the one-dimensional and two-dimensional situations, we now switch from scalar to vector notation. In this way, a contour is defined as that set of vector points $(\bar{x})$ for which it holds that $f_{t}(\bar{x})$ is a constant. We now come to the question of which constant should be selected. If a point is situated such that no clustering is possible (for instance, an isolated point), then this point virtually is an infinitely small cluster at its own position. This situation can be described by taking the threshold value to be the maximum height of the partial density function. So, the contour in question is now defined as that set of points $(\bar{x})$ for which it holds that $f_{t}(\bar{x})=f_{0}$, with $f_{0}$ being the peak value of the spread function.

We now proceed to the case of a set of $n$ dots, labeled $\mathrm{p}_{1}, \mathrm{p}_{2}, \ldots, \mathrm{p}_{\mathrm{n}}$, with positions $\overline{\mathrm{x}}_{1}, \overline{\mathrm{x}}_{2}, \ldots, \overline{\mathrm{x}}_{\mathrm{n}}$, respectively. A second assumption is made in these cases. That is, with the function $f_{p_{i}}(\bar{x})$ of a particular dot $p_{i}$ in the set, the dispersion equals half the distance of that element to its nearest neighbor, $s_{i}=1 / 2 d\left(\bar{x}_{i}, \bar{x}_{j}\right)$, with $\bar{x}_{j}$ equaling the position of $p_{j}$, the nearest neighbor of $\mathrm{p}_{\mathrm{i}}$. In this way, the strength of a point's influence upon its surroundings depends on the distance to its nearest neighboring point. Once the dispersion value, and therefore the distribution function, has been established for each dot of the set, all partial distribution functions are summed:

$$
f_{t}(\bar{x})=\sum_{i=1}^{i=n} f_{p_{i}}(\bar{x}) .
$$

The resulting landscape of mountains and valleys can be seen to be quite similar to Marr's (Note 1) primary 


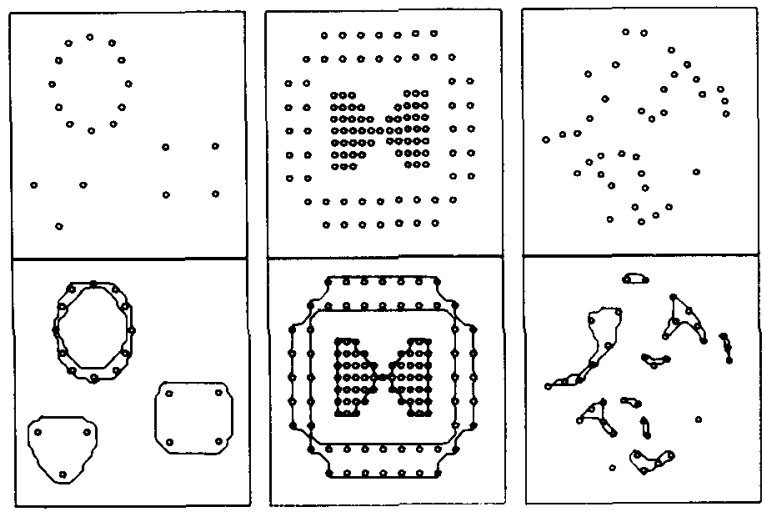

Figure 3. Three examples of dot figures that were subjected to CODE (top). Below are the resulting clusters within the dot patterns.

sketch. The contour specified by the relation $f_{t}(\bar{x})=f_{0}$ is computed along similar lines to the $n=2$ case. A cluster is then defined as the subset of all dots that lie precisely on, or within, that particular contour. It is important to notice that clusters are formed in regions in which dots are mutually near neighbors rather than simply near neighbors. CODE considers only relative proximity between dots, rather than absolute distances. As a consequence, CODE is invariant under similarity transformations (translation, rotation, and changes of size). Also, CODE is insensitive to the order in which the points of a figure are scrutinized. The proposed method is illustrated by a few examples in Figure 3.

The following experiment examined whether CODEdetermined clustering corresponded well with human observers' perceptions. If CODE is a good predictor of human performance, it then makes sense to apply it in examining the influence of grouping on processing of visual numerosity.

\section{EXPERIMENT 1}

\section{Method}

Subjects. Five undergraduate psychology students (four males, one female), who were naive to the experimental task, were paid for their participation in the experiment.

Stimuli. Seventy-two dot figures were constructed. Each figure differed both in number $(n=14-23)$ and arrangement of dots. Seven different arrangements were used, except for $n=22$ and $n=23$, for which there were eight arrangements.

For each number of $n$, there was one configuration consisting of 1 large cluster $\left(n_{g}=1\right)$, one configuration of 2 clusters $\left(n_{g}=2\right)$, and so on, up to one configuration properly segmented into $n_{g}=7$ (or $n_{g}=8$ for $n=22$ and $n=23$ ) distinct clusters. Care was taken that different clusters within one figure contain about the same number of dots. Figures were constructed by assigning dots to different cell positions of a 15 by 15 matrix. A dot was always placed randomly in the center of a cell matrix or just above, below, or next to that position for a distance of .2 of the cell width. The objective criteria for the clustering within each stimulus were established by CODE. Figure 4 depicts eight of the test stimuli with the same number of dots $(n=22)$, but with different arrangements.

In addition to these test stimuli, 18 fillers were constructed that depicted randomly arranged dot figures. Ten of them $(n=14-23)$ were used to make the appearance of equal group sizes within one stimulus less obvious. The other eight figures were used to avoid possible range effects. Their numbers of dots were in the ranges 10-13 and 24-27.

Procedure. Participants were tested individually in a quiet laboratory room. The stimuli were presented on a $27 \times 20 \mathrm{~cm}$ video monitor situated approximately $75 \mathrm{~cm}$ from the participant and at eye level. The dot diameter was $2 \mathrm{~mm}$. The size of the stimulus was made to fit as well as possible within an area of $10 \times 10 \mathrm{~cm}$, with the midpoint of this area in the middle of the monitor. Thus, the stimuli were viewed at about an 8-deg angle. Each stimulus was presented for a fixed duration of $100 \mathrm{msec}$. Presentation order was random, except that the first three stimuli were always filler stimuli. The task was self-paced; a stimulus appeared on the screen 1,000 msec after the participant had pushed a button. Participants were instructed to attend to the number of groups in each stimulus and to report this number orally. The experimenter recorded the participants' responses. Following 88 trials, the participants rested a few minutes. The next stimulus sequence contained the same stimuli presented in a different random order and in a different orientation. Orientation was varied either by rotating the full configuration $90 \mathrm{deg}$ or by mirroring along the vertical or horizontal axis. All participants completed five or six series of 88 stimulus presentations each. The experiment was run under the control of a PDP-11/45 computer.

\section{Results}

Responses of all participants given with respect to a particular $n_{g}$ value, regardless of the numerosity $(n)$ of the stimulus, were classified into one category. This categorization was done for each of the eight cluster levels $\left(\mathrm{n}_{\mathrm{g}}=1\right.$ through $\left.\mathrm{n}_{\mathrm{g}}=8\right)$. Table 1 summarizes
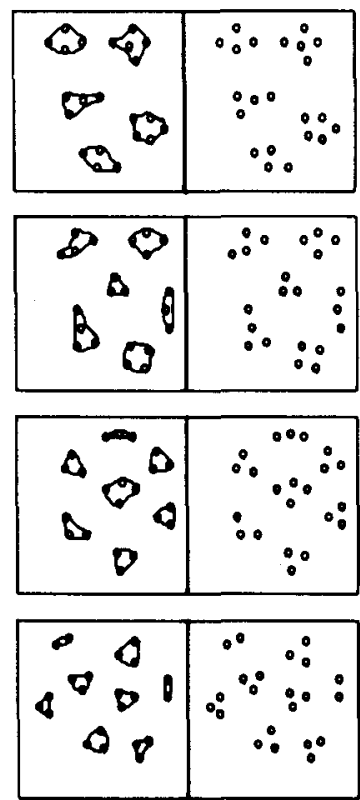
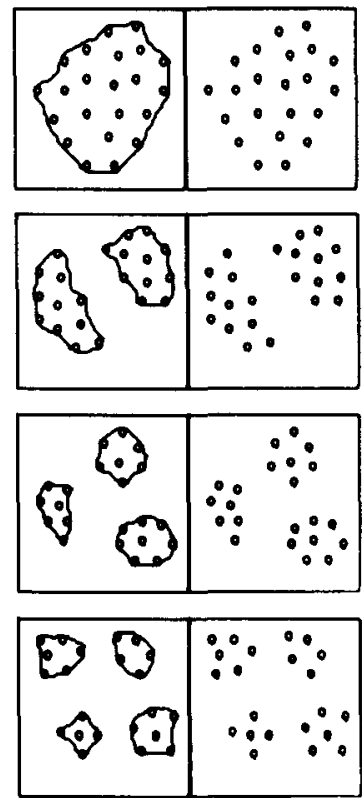

Figure 4. Eight test stimuli, with the same number of dots $\left(n_{d}=22\right)$, but differently arranged $\left(n_{g}=1-8\right)$. 
Table 1

Reported Numbers of Groups (Subjective) vs. Presented Number of Clusters (Objective)

\begin{tabular}{rrrrrrrrr}
\hline & \multicolumn{10}{c}{$\mathrm{R}$} \\
\cline { 2 - 9 } & \multicolumn{1}{c}{1} & \multicolumn{1}{c}{2} & \multicolumn{1}{c}{3} & \multicolumn{1}{c}{4} & 5 & 6 & 7 & 8 \\
\hline 1 & 155 & 3 & 1 & 1 & 0 & 0 & 0 & 0 \\
2 & 5 & 134 & 9 & 11 & 1 & 0 & 0 & 0 \\
3 & 0 & 1 & 140 & 15 & 4 & 0 & 0 & 0 \\
4 & 0 & 1 & 4 & 151 & 4 & 0 & 0 & 0 \\
5 & 1 & 3 & 2 & 9 & 135 & 9 & 1 & 0 \\
6 & 6 & 8 & 5 & 7 & 25 & 104 & 5 & 0 \\
7 & 5 & 4 & 2 & 1 & 4 & 44 & 96 & 4 \\
8 & 2 & 1 & 0 & 1 & 2 & 8 & 15 & 3 \\
\hline
\end{tabular}

Note $-R=$ reported number of groups; $P=$ presented number of clusters.

actually reported numbers of groups per $n_{g}$ category. Figure 5 shows the percentages of responses that were in agreement with the CODE-predicted clusters for each category. The histogram shows high percentages $(>80 \%)$ of CODE-predicted clusters for the levels $n_{g}=1$ through $\mathrm{n}_{\mathrm{g}}=5$. These percentages declined rapidly for the larger $\mathrm{n}_{\mathrm{g}}$ values.

\section{Discussion}

The relationship between CODE-predicted clusters and humans' perceptions of groups was examined by asking participants to estimate the number of groups of dots in a stimulus. Participants were given only $100 \mathrm{msec}$ to compose their estimates, a period that is evidently too short for successive one-by-one counting of groups. Hence, subitizing is the only means available to perform the task. As is well-known, subitizing has an upper limit of about five dots. This limitation is expressed dramatically in the data. For cluster values smaller than $n_{g}=6$, the reported numbers of groups corresponded well with CODE predictions. The rapid decline in correspondence for larger $n_{\mathrm{g}}$ values most probably reflects the difficulty of subitizing these larger numbers. In order to overcome the problems of noisy results with larger $n_{g}$ values, a latency experiment might seem a better experimental procedure. In this case, however, we cannot prevent participants' applying sophisticated cognitive strategies that might reflect more than proximity-based percepts.

From the given data, we conclude that CODE is robust enough to give a formal account of how grouping by relative proximity takes place in dot patterns.

In the next section, we will apply CODE to the analysis of how subjective grouping influences the processing of visual number of sets of dots.

Effects of grouping on the processing of visual number. Before describing how grouping affects the abstraction of number from figures with more than 10 dots, it is useful to distinguish between two grouping conditions. In one condition (called "small groups"), all distinct groups in a particular figure have about the same number of dots and these numbers are all within the subitizing range. An example is a configuration of 24 dots consisting of six groups of 4 dots each. In the second condition (labeled "large groups"), the various groups also contain the same number of dots, but their number now exceeds the subitizing span. This condition is exemplified by the case of 24 dots segmented into three groups of 8 dots each.

Response latencies to correct number responses provide an appropriate way to measure differential effects of groupability on the processing of visual number. The processing time for stimuli under the small-group condition is assumed to be composed of the following constituents: (1) a constant amount of time for the perceptual segmentation of a dot figure into groups, (2) time needed for subitizing the number of dots within a group, (3) time to compute the running sum of the partial results, and (4) a constant amount of time for the overt verbalization of the response. Thus, response latency is a function of both total number of dots, $n_{d}$, and the number of groups, $\mathrm{n}_{\mathrm{g}}$, and is expressed formally as:

$$
\operatorname{RT}\left(\mathrm{n}_{\mathrm{d}}, \mathrm{n}_{\mathrm{g}}\right)=\mathrm{b}_{0}+\mathrm{n}_{\mathrm{d}} \mathrm{b}_{1}+\left(\mathrm{n}_{\mathrm{g}}-1\right) \mathrm{b}_{2},
$$

in which $b_{0}$ stands for grouping and motor response time, $b_{1}$ stands for the time per dot consumed by the subitizing process, $b_{2}$ stands for the time to switch from one group to another and to compute the running sum, and RT stands for response latency. The number of these last operations equals $n_{g}-1$. Since we are interested here in response latency as a function of group size $\left(g_{n}\right)$, and since average group size $\left(\left\langle g_{n}\right\rangle\right)$ equals $\mathrm{n}_{\mathrm{d}} / \mathrm{n}_{\mathrm{g}}$, Equation 1 can be rewritten as:

$$
\operatorname{RT}\left(\mathrm{n}_{\mathrm{d}},\left\langle\mathrm{g}_{\mathrm{n}}\right)\right)=\mathrm{b}_{0}+\mathrm{n}_{\mathrm{d}} \mathrm{b}_{1}+\left[\left(\mathrm{n}_{\mathrm{d}} /\left\langle\mathrm{g}_{\mathrm{n}}\right\rangle\right)-1\right] \mathrm{b}_{2} .
$$

Dividing RT by $n_{d}$ gives us the reaction time per single dot:

$\operatorname{RT}\left(\mathrm{n}_{\mathrm{d}},\left\langle\mathrm{gn}_{n}\right\rangle\right) / \mathrm{n}_{\mathrm{d}}=b_{1}+\left[\left(\mathrm{b}_{0}-\mathrm{b}_{2}\right) / \mathrm{n}_{\mathrm{d}}\right]+\left(\mathrm{b}_{2} /\left\langle\mathrm{g}_{\mathrm{n}}\right\rangle\right)$.

The value of $b_{2}$ is about $400 \mathrm{msec}$ (Vos \& van Oeffelen, Note 3). The exposure duration in Experiment 1 was $100 \mathrm{msec}$, which was sufficient to allow perception of

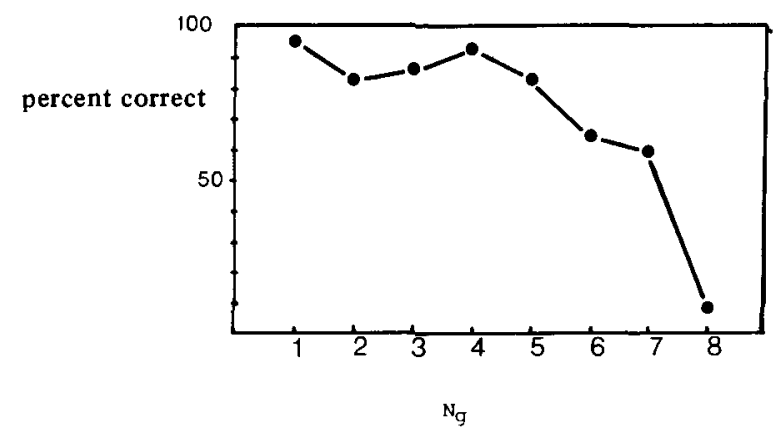

Figure 5. The percentages of responses that were in agreement with CODE-predicted clusters for each category $\mathbf{n}_{\mathbf{g}}$. 
groups. Eriksen and Collins (1968) reported a useful duration of dots in iconic memory of about $100 \mathrm{msec}$. So, $b_{0}$ amounts to about $200 \mathrm{msec}$. In addition to the fact that $n_{d}$ is much larger than group size $\left\langle g_{n}\right\rangle$, we can ignore the second term in Equation 3 with respect to the third term and we may safely approximate Equation 3 by an expression for reaction time per dot that is a function of $\left\langle\mathrm{g}_{\mathrm{n}}\right\rangle$ only:

$$
\operatorname{RT}\left(\left\langle g_{n}\right\rangle\right) / n_{d}=b_{1}^{\prime}+\left(b_{2} /\left\langle g_{n}\right\rangle\right) .
$$

Equation 4 says that, under the small-group condition, the reaction time per dot is a hyperbolic function of average group size.

We consider next the processing of dot figures in a large group. Another quantification strategy has to be followed when the initial proximity-based groupings are too large to be subitized. A plausible strategy is to first count one by one the dots within one group, after which the process of enumeration (see Beckwith \& Restle, 1966 ) is continued with the dots of a second group, and so on. The only advantage of groupability (large groups) might be that it facilitates the discrimination between dots already counted and those still to be counted. Earlier studies on the processing of visual number (Aoki, 1977; Beckwith \& Restle, 1966; Klahr, 1973) indicate that counting one by one requires about $300-400 \mathrm{msec}$ for a dot to be enumerated; hence, response latency should be a linear function of $n_{d}$ :

$$
\operatorname{RT}\left(\mathrm{n}_{\mathrm{d}}\right)=\mathrm{A}+\mathrm{Bn_{ \textrm {d } }} \text {. }
$$

However, the same studies also suggest that a subject might still form groups consisting of two, three, or perhaps even more dots during the process of counting. If so, Equation 1, proposed for the case of small groups can also be applied to the present condition. Nevertheless, an extra component has to be introduced representing the large proximity-based groups. The equation then reads as follows:

$$
\operatorname{RT}\left(\mathrm{n}_{\mathrm{d}}, \mathrm{n}_{\mathrm{s}}\right)=\mathrm{b}_{0}+\mathrm{n}_{\mathrm{d}} \mathrm{b}_{1}+\left(\mathrm{n}_{\mathrm{s}}-1\right) \mathrm{b}_{2}+\mathrm{b}_{3}\left(\mathrm{n}_{\mathrm{d}}, \mathrm{n}_{\mathrm{s}}\right) .
$$

The number of subgroups is now represented by $n_{s}$, and $b_{3}\left(n_{d}, n_{s}\right)$ stands for the time to detect subgroups during the counting process. Since we do not know how large the identified subgroups are and do not know, therefore, how many subgroups there are, we perform some averaging with respect to the grouping-dependent terms. We assume that, averaged over a large set of dot figures under the large-group condition, a subject detects a fraction $E(1)$ of ones, a fraction $E(2)$ of twos, and so on, up to a fraction $E(S)$ of subgroups the size of which equals the upper limit (S) of the span of subitizing. In that case, the mean number of subgroups $\left(n_{s}\right)$ should be

$$
\sum_{i=1}^{i=S} E(i) \frac{n_{d}}{i}=\left\{\sum_{i=1}^{i=S}[E(i) / i]\right\} n_{d}
$$

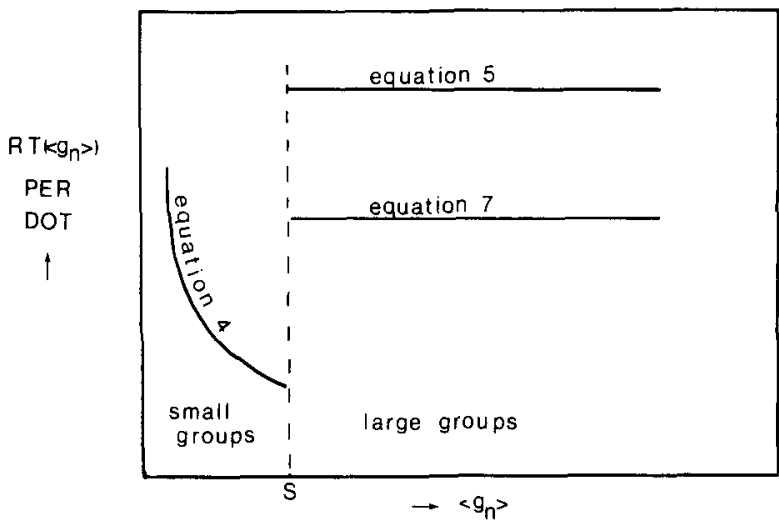

Figure 6. Expected reaction time per dot schematized as a function of initial (proximity-based) groupability $\left\langle g_{n}\right\rangle$ (number of dots within a group).

Further, we assume that the average time to perform such subgrouping increases proportionally with increasing total number of dots, $n_{d}$, so that $\left\langle b_{3}\left(n_{d}, n_{s}\right)\right\rangle=b_{3}^{\prime} n_{d}$, of which $b_{3}^{\prime}$ is a constant. With these assumptions made, we can rewrite Equation 6 as follows:

$$
\begin{aligned}
& \left\langle\operatorname{RT}\left(n_{d}, n_{s}\right)\right\rangle= \\
& b_{0}+n_{d} b_{1}+\left\{\sum_{i=S}^{i=S}[E(i) / i]\right\}\left(n_{d}-1\right) b_{2}+b_{3}^{\prime} n_{d} .
\end{aligned}
$$

The factor

$$
\left\{\sum_{\mathrm{i}=1}^{\mathrm{i}=\mathrm{S}}[\mathrm{E}(\mathrm{i}) / \mathrm{i}]\right\}
$$

clearly is a constant, so all terms in Equation 7 are either constants or linear with respect to $n_{d}$. Therefore, we can present Equation 7 in a much simpler form:

$$
\left\langle\operatorname{RT}\left(\mathrm{n}_{\mathrm{d}}\right)\right\rangle=\mathrm{A}^{\prime}+\mathrm{B}^{\prime} \mathrm{n}_{\mathrm{d}} .
$$

Figure 6 schematizes the expected reaction time per dot as a function of initial (proximity-based) groupability. For dot figures with group sizes within the subitizing range, reaction time per dot is a hyperbolic function of average group size $\left\langle\mathrm{g}_{\mathrm{n}}\right\rangle$. For dot figures with larger group sizes, reaction time per dot should be independent of group size $\left\langle\mathrm{g}_{n}\right\rangle$.

\section{EXPERIMENT 2}

\section{Method}

Subjects. Eight undergraduate psychology students (seven males, one female) were paid for their participation in the experiment. Five of them had also participated in Experiment 1.

Stimuli. The same set of stimuli used in Experiment 1 was also used in the present experiment.

Procedure. The presentation of the stimuli differed from that of Experiment 1 in that each stimulus appeared on the monitor immediately after the participant had pushed a button. The stim- 
ulus remained visible until the response, mediated by a microphone (Sennheiser headset), had surpassed a previously selected critical level. Participants were asked to report the number of dots as quickly and accurately as possible. Latencies were registered automatically. The experimenter, who had a list of stimulus specifications, scored each response according to whether it was correct or incorrect and then stored it into the computer. Whenever a participant committed an error, she/he received immediate feedback ("wrong") from the experimenter. Each sequence of 88 stimuli contained the same stimuli, presented in a different random order and in a different orientation. As in Experiment 1, orientation was changed either by rotating the full configuration $90 \mathrm{deg}$ or by mirroring along the vertical or horizontal axis.

Each participant completed 12-16 sequences in three sessions spread out over 2 or 3 subsequent days. Each session lasted about $90 \mathrm{~min}$.

\section{Results}

No more than $5 \%$ of participants' responses were incorrect. The statistical analysis was restricted to correct responses given to the 72 test stimuli.

The mean and standard deviation of a minimum of 12 and a maximum of 16 repeatedly measured latencies were computed for each subject and stimulus. The standard deviations typically were on the order of $7 \%$ $12 \%$ and never reached values larger than $20 \%$ of the means. The mean reaction times obtained over all participants are shown in Figure $7 \mathrm{a}$ as a function of $n_{d}$ for each of the seven (or eight, for $n_{d}=22$ and 23) different $\mathrm{n}_{\mathrm{g}}$ conditions.

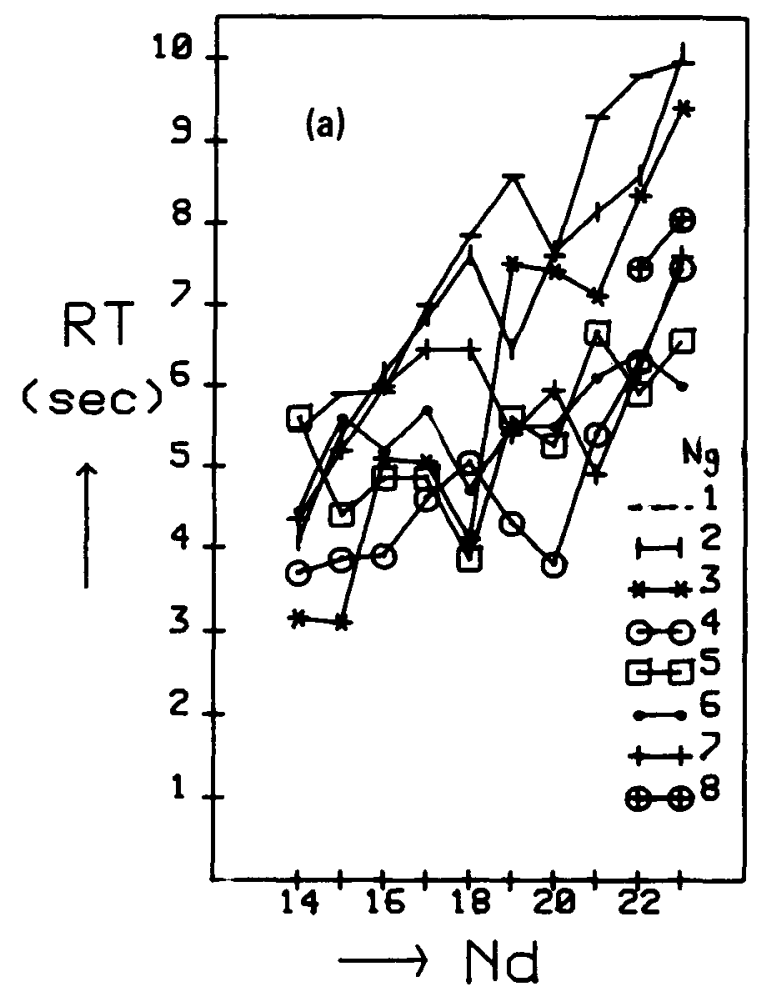

Figure $7 \mathrm{~b}$ shows the relation between reaction time and $n_{d}$ in a more traditional way, in which averaged values over different $n_{g}$ conditions belonging to the same $\mathrm{n}_{\mathrm{d}}$ value are plotted. The maximum and minimum values of response latency depicted in Figure 7a, regardless of groupability, are also plotted, as are reaction times on randomly arranged dot patterns derived from Jensen, Reese, and Reese (1950). Figure $7 \mathrm{~b}$ shows that latencies roughly tended to increase with absolute number, regardless of dot arrangements.

In order to minimize effects of interindividual dif. ferences in latencies, the averaged data of each subject were normalized. Figure 8 presents the normalized mean reaction times as a function of mean group size $\left\langle g_{n}\right\rangle$ for each different value of $n_{d}$. All 10 curves are patterned largely such that (1) for group sizes up to about five dots, latencies tended to decrease, (2) from $\left\langle\mathrm{g}_{\mathrm{n}}\right\rangle=6$ onward, latencies rather abruptly jumped back to about the level obtained for $\left\langle g_{n}\right\rangle=2$, and (3) for larger values of $\left\langle g_{n}\right\rangle$, there was a weak latency increase.

Dividing the various latencies by the corresponding values of $n_{d}$, the overall means of processing time per dot were obtained as a function of $\left\langle g_{n}\right\rangle$. From value $\left\langle\mathrm{g}_{\mathrm{n}}\right\rangle=2$ on, latencies per dot were subjected to a hyperbolic fit in accordance with the predictions formulated in Equation 1. Good fits were found for all latencies up to and including level $\left\langle\mathrm{g}_{n}\right\rangle=5\left[\mathrm{RT} / \mathrm{n}_{\mathrm{d}}=\left(1 / \mathrm{g}_{\mathrm{n}}\right\rangle \mathrm{X}\right.$ $466)+12 \mathrm{msec} / \mathrm{dot}, \mathrm{p}=.86]$. Taking the line of Equa-

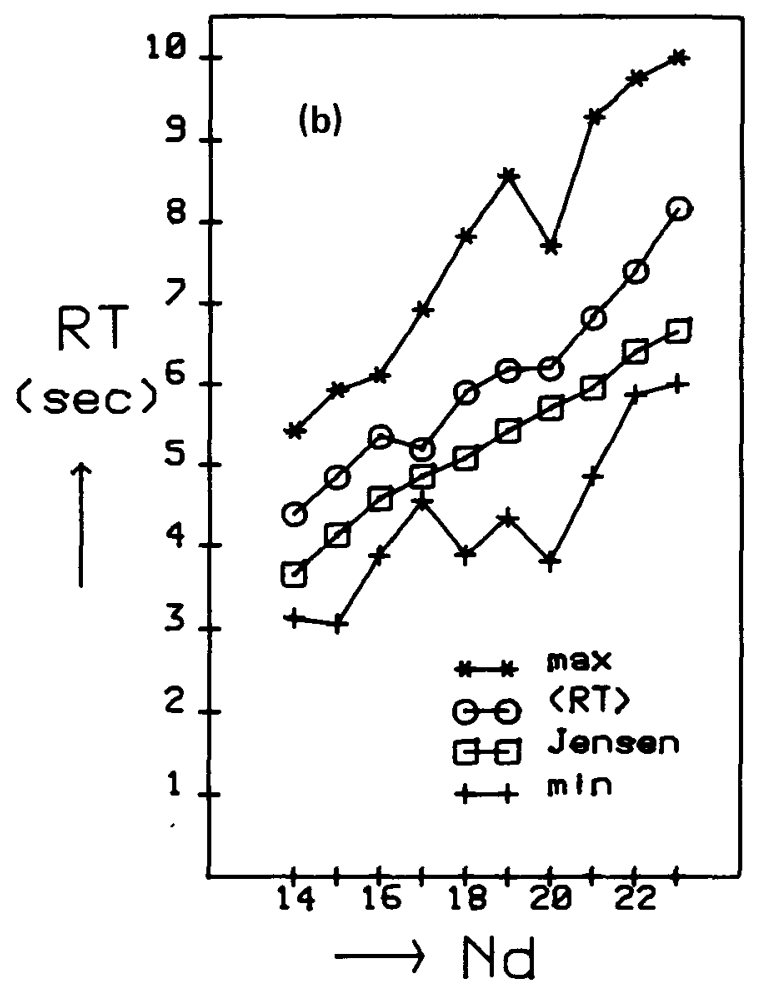

Figure 7. (a) For each of the seven (or eight, for $n_{d}=22$ and 23) different $n_{g}$ conditions (number of groups), the mean reaction times are plotted as a function of $n_{d}$, the total number of dots. (b) The mean reaction times over all $n_{\mathfrak{g}}$ conditions are plotted as a function of $n_{d}$. The maximum and minimum values of reaction time, depicted in Figure 7a, regardless of groupability, are also plotted, as are reaction times on randomly arranged dot patterns derived from Jensen et al. (1950). 

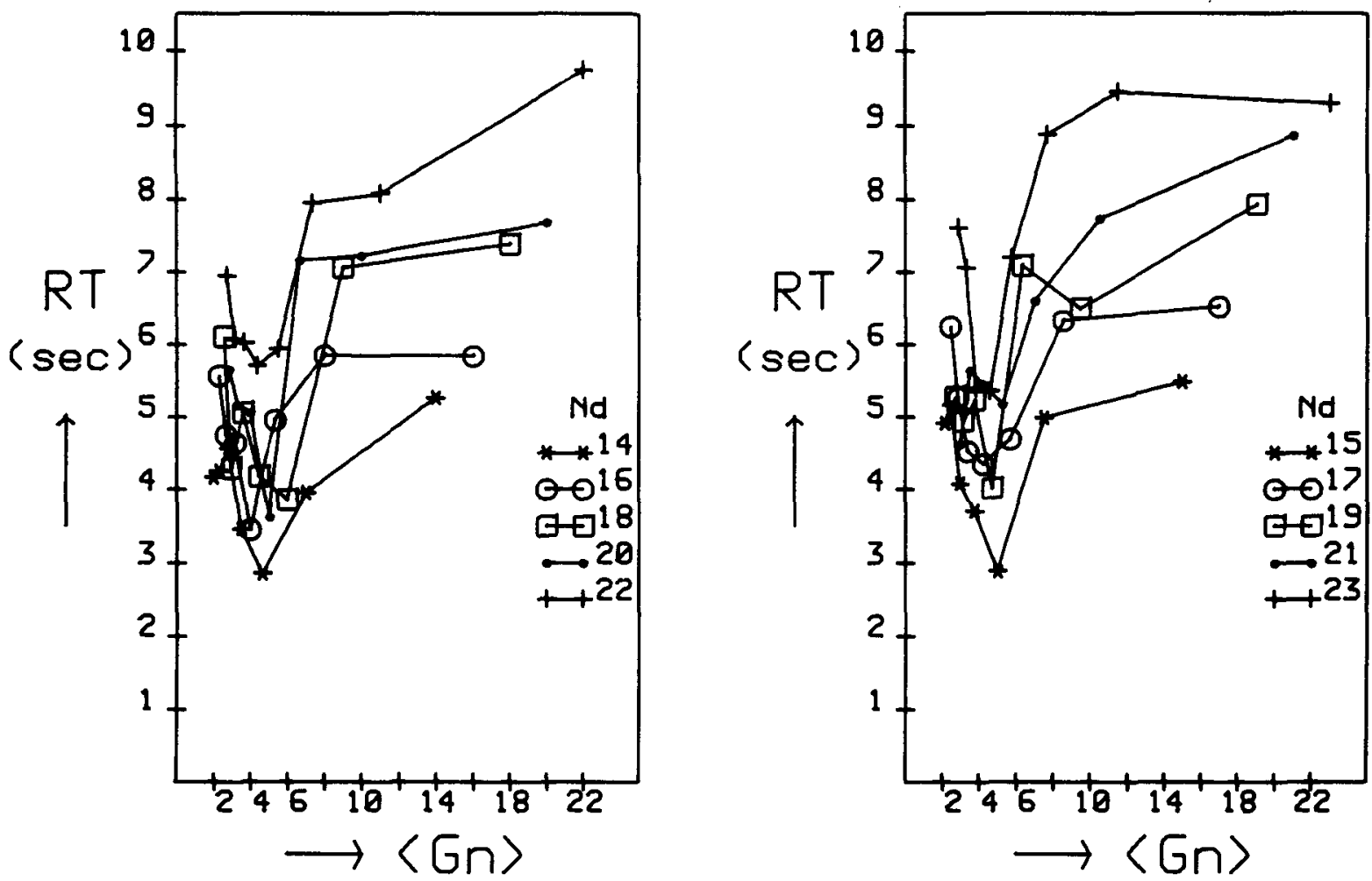

Figure 8. The normalized mean reaction times are plotted as a function of $\left(\mathrm{g}_{\mathbf{n}}\right\rangle$ for each number $\mathrm{n}_{\mathbf{d}}$.

tions 5 and 8, a linear fit was carried out on latencies at the higher $\left\langle g_{n}\right\rangle$ values to be reckoned from $\left\langle g_{n}\right\rangle=23$ downward. A good fit $(\mathrm{p}=.85)$ was found in the interval $6 \leqslant\left\langle\mathrm{~g}_{n}\right\rangle \leqslant 23\left(\mathrm{RT} / \mathrm{n}_{\mathrm{d}}=4\left(\mathrm{~g}_{\mathrm{n}}\right\rangle+320 \mathrm{msec} / \mathrm{dot}\right)$. The goodness of fit for the hyperbolic part, as well as for the linear one, deteriorated considerably when latencies in the interval $5<\left\langle\mathrm{g}_{n}\right\rangle<6$ (shaded area in Figure 9) were also considered.

\section{Discussion and Conclusions}

The data of Experiment 2 supported our theoretical description of how ascertaining the size of a large set of dots is affected by its patterning. Latencies for one and the same set size appeared to follow two disjoint trends, the empirically derived transition point being the passage from (averaged) group size $\left\langle g_{n}\right\rangle=5$ to $\left\langle g_{n}\right\rangle=6$. Under the small-group conditions $\left(2 \leqslant\left\langle g_{n}\right\rangle \leqslant 5\right)$, number was apparently ascertained by a combined strategy of subitizing the groups and adding the results to a running sum. The hyperbolically shaped drop from approximately $350 \mathrm{msec} / \mathrm{dot}$ when $\left\langle\mathrm{g}_{\mathrm{n}}\right\rangle=2$ to a minimum of roughly $200 \mathrm{msec} / \mathrm{dot}$ when $\left\langle\mathrm{g}_{\mathrm{n}}\right\rangle=5$ confirmed our hypothesis that under small-group conditions, the processing time for the whole set decreases with a reduction in the number of adding operations. The picture of the data obtained to the right of the transition point was more complex than predicted. In accordance with the model, those data were appropriately fitted by a straight line. The slope of the line, however, was not zero but slightly positive. The increase of processing

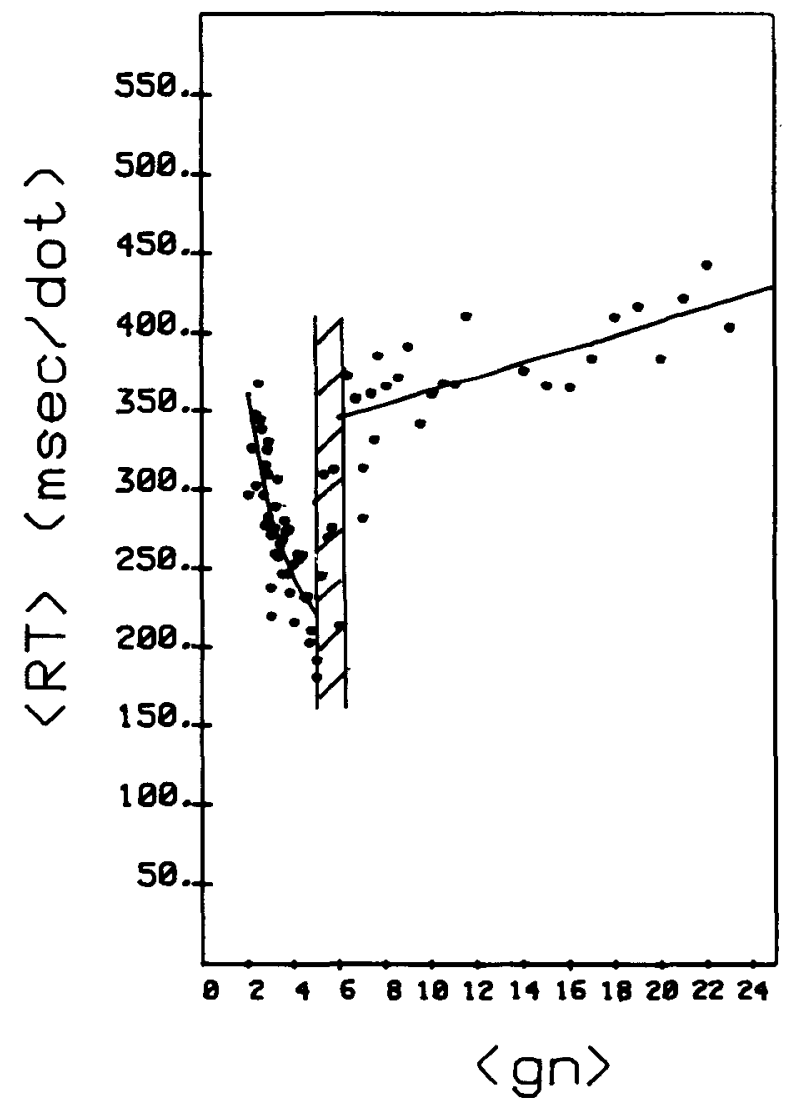

Figure 9. The normalized mean reaction times per dot are plotted as a function of $\langle\mathrm{g} n\rangle$. 
time per dot with larger $\left\langle g_{n}\right\rangle$ values was probably due to increased effects of lateral interference in progressively larger groups of dots, a factor not considered in our initial model. Under the large-group conditions, the data suggested that the dots were counted in (sub)groups rather than one by one. This conclusion is supported by the findings that mean processing time per dot was in the same order of magnitude in the $\left\langle\mathrm{g}_{n}\right\rangle=6$ to $\left\langle\mathrm{g}_{\mathrm{n}}\right\rangle=10$ conditions as in the $\left\langle\mathrm{g}_{\mathrm{n}}\right\rangle=2$ and $\left\langle\mathrm{g}_{\mathrm{n}}\right\rangle=3$ conditions. In other words, it seems that the perceiver splits the larger groups into subgroups of two or three dots (subjective grouping) and adds the results together in largely the same way as it is done under the small-group conditions. It is still unclear how subjective grouping takes place in the absence of proximity cues. One possibility is that counting in twos and threes is a common routine for normal adults. For example, the chant "two, four, six, ..."' is a slightly overlearned one for most of them. Another possibility is that the perceiver recognized patterns. Neisser (1967) pointed to the fact that three randomly positioned dots nearly always make a triangle and it often is also easy to detect a quadrangle in four neighboring dots. Extending the search for meaningful patterns beyond clusters of three dots may increase, for example, the risk of lateral interference, with concomitantly greater errors. This speculation warrants further research. One might, for example, present large-group stimuli and ask the subject to ascertain the number of dots under different counting instructions, such as counting in twos, threes, fours, and so forth. If our speculation is correct, latencies and errors should abruptly increase when counting must be done in fours or more.

While the idea that the processing of visual number is affected by patterning of the objects is not new (cf. Aoki, 1977; Atkinson et al., 1976a, 1976b; Beckwith \& Restle, 1966; Bourdon, 1908; Freeman, 1912; Hamilton, 1865), our study is the first attempt to account for the number-pattern interaction on the basis of a quantitative theory. Patterning was exclusively dealt with at its most elementary level of grouping by proximity. Indeed, it is difficult to imagine even random configurations of dots that cannot be perceptually organized into distinct groups of relatively proximal dots. Consequently, the first step of our approach was to develop an algorithm to simulate how a human perceiver realizes the patterning in question. We think that CODE has been proved to be a useful tool for the quantitative specification of dot groupability in random patterns. Its applicability might be extended to other related problem fields, such as judgments of areas bounded by subjective contour.

\section{REFERENCE NOTES}

1. Marr, D. Analyzing natural images, a computational theory of vision (AI Memo 334). Cambridge: M.I.T. Artificial Intelligence Laboratory, 1975.

2. Zucker, W., Rosenfeld, A., \& Davis, L. S. General purpose models: Expectations about the unexpected (Technical Report 347). College Park: University of Maryland, Computer Center, 1975. 3. Vos, P. G., \& van Oeffelen, M. P. Arithmetical operations with dot patterns: A chronometric analysis (Internal Report,ONO6). Nijmegen: University of Nijmegen, 1982.

\section{REFERENCES}

Aokı, T. On the counting process of patterned dots. Tohuku Psychologica Folia, 1977, 36(1-4), 15-22.

Atkinson, J., Cambell, F. W., \& Francis, M. R. The dependence of the visual numerosity limit on orientation, colour, and grouping in the stimulus. Perception, 1976, 5, 335-342. (a)

Atkinson, J., Cambell, F. W., \& Francis, M. R. The magic number $4 \pm 0$ : A new look at visual numerosity judgements. Perception, 1976, 5, 327-334. (b)

Beckwith, M., \& Restre, F. Process of enumeration. Psychological Review, 1966, 73, 437-444.

Bourdon, B. Sur le temps nécessaire pour nominer les nombres. Revue Philosophique de la France et de l'Etranger, 1908, 33, 426-431.

Eriksen, C. W., \& Collins, J. F. Sensory traces versus the psychological moment in the temporal organization of form. Journal of Experimental Psychology, 1968, 77, 376-382.

Freeman, F. N. Grouped objects as a concrete basis for the number idea. Elementary School Teacher, 1912, 12, 306-314.

Hamilton, W. Lectures on metaphysics and logic. London: Blackwood, 1865.

Jensen, E. M., Reese, E. P., \& Reese, T. W. The subitizing and counting of visually presented fields of dots. Journal of Psychology, 1950, 30, 363-392.

Kaufman, E. L., Lord, M. W., Reese, T. W., \& Volkman, J. The discrimination of visual number. American Journal of Psychology, 1949, 62, 498-525.

KLAHR, D. A production system for counting, subitizing, and adding. In W. G. Chase (Ed.), Visual information processing. New York: Academic Press, 1973.

Klahr, D., \& Wallace, J. G. Cognitive development: An information processing view. New York: Wiley, 1976.

NE1sser, U. Cognitive psychology. New York: Appleton-CenturyCrofts, 1967.

Woodworth, R. S., \& SchlosBerg, H. Experimental psychology. New York: Holt, Rinehart \& Winston, 1954.

ZusNe, L. Visual perception of form. New York: Academic Press, 1970.

(Received for publication November 5, 1981; revision accepted February 10, 1982.) 